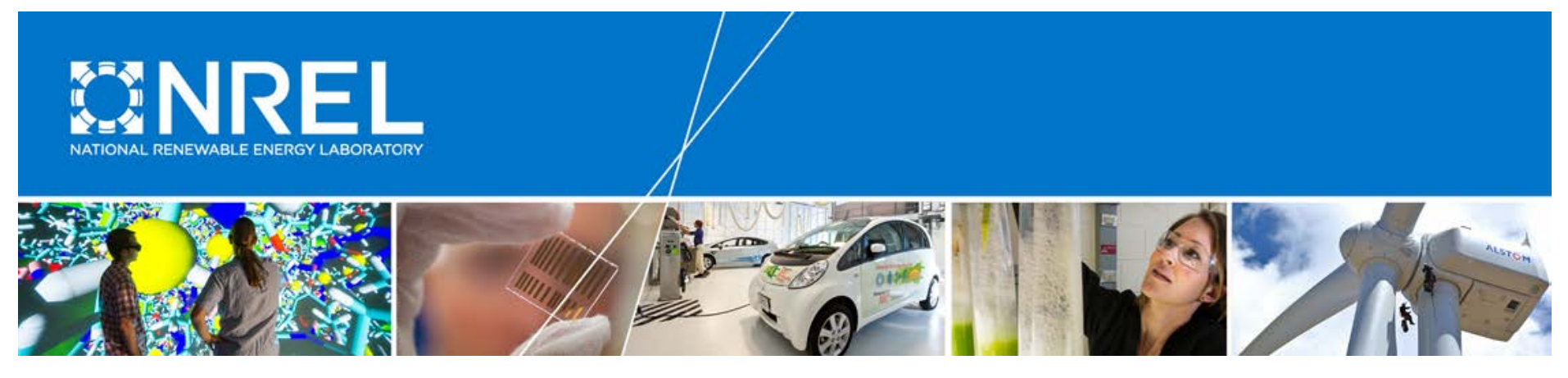

\title{
Primary Frequency Response with Aggregated DERs
}

\section{Preprint}

Swaroop S. Guggilam and Sairaj V. Dhople University of Minnesota

Changhong Zhao and Emiliano Dall'Anese National Renewable Energy Laboratory

\section{Yu Christine Chen}

University of British Columbia

To be presented at the 2017 American Control Conference Seattle, Washington

May 24-26, 2017

NREL is a national laboratory of the U.S. Department of Energy Office of Energy Efficiency \& Renewable Energy Operated by the Alliance for Sustainable Energy, LLC

This report is available at no cost from the National Renewable Energy Laboratory (NREL) at www.nrel.gov/publications.

\section{Conference Paper}

NREL/CP-5D00-67883

March 2017 


\section{NOTICE}

The submitted manuscript has been offered by an employee of the Alliance for Sustainable Energy, LLC (Alliance), a contractor of the US Government under Contract No. DE-AC36-08GO28308. Accordingly, the US Government and Alliance retain a nonexclusive royalty-free license to publish or reproduce the published form of this contribution, or allow others to do so, for US Government purposes.

This report was prepared as an account of work sponsored by an agency of the United States government. Neither the United States government nor any agency thereof, nor any of their employees, makes any warranty, express or implied, or assumes any legal liability or responsibility for the accuracy, completeness, or usefulness of any information, apparatus, product, or process disclosed, or represents that its use would not infringe privately owned rights. Reference herein to any specific commercial product, process, or service by trade name, trademark, manufacturer, or otherwise does not necessarily constitute or imply its endorsement, recommendation, or favoring by the United States government or any agency thereof. The views and opinions of authors expressed herein do not necessarily state or reflect those of the United States government or any agency thereof.

This report is available at no cost from the National Renewable Energy Laboratory (NREL) at www.nrel.gov/publications.

Available electronically at SciTech Connect http:/www.osti.gov/scitech

Available for a processing fee to U.S. Department of Energy and its contractors, in paper, from:

U.S. Department of Energy

Office of Scientific and Technical Information

P.O. Box 62

Oak Ridge, TN 37831-0062

OSTI http://www.osti.gov

Phone: 865.576.8401

Fax: 865.576.5728

Email: reports@osti.gov

Available for sale to the public, in paper, from:

U.S. Department of Commerce

National Technical Information Service

5301 Shawnee Road

Alexandria, VA 22312

NTIS http://www.ntis.gov

Phone: 800.553 .6847 or 703.605 .6000

Fax: 703.605.6900

Email: orders@ntis.gov 


\title{
Primary Frequency Response with Aggregated DERs
}

\author{
Swaroop S. Guggilam, Changhong Zhao, Emiliano Dall'Anese, Yu Christine Chen, and Sairaj V. Dhople
}

\begin{abstract}
Power networks have to withstand a variety of disturbances that affect system frequency, and the problem is compounded with the increasing integration of intermittent renewable generation. Following a large-signal generation or load disturbance, system frequency is arrested leveraging primary frequency control provided by governor action in synchronous generators. In this work, we propose a framework for distributed energy resources (DERs) deployed in distribution networks to provide (supplemental) primary frequency response. Particularly, we demonstrate how power-frequency droop slopes for individual DERs can be designed so that the distribution feeder presents a guaranteed frequency-regulation characteristic at the feeder head. Furthermore, the droop slopes are engineered such that injections of individual DERs conform to a well-defined fairness objective that does not penalize them for their location on the distribution feeder. Time-domain simulations for an illustrative network composed of a combined transmission network and distribution network with frequencyresponsive DERs are provided to validate the approach.
\end{abstract}

\section{INTRODUCTION}

Traditional approaches for regulating and maintaining system frequency in power transmission systems leverage inertial response, primary frequency response, and automatic generation control provided by synchronous generators. In the future, on the other hand, distributed energy resources (DERs) at both utility level and in commercial/residential settings are envisioned to complement traditional generationside capabilities at multiple time scales to aid frequency regulation and restoration due to disturbances arising from unexpected contingencies and intermittent renewable sources [1][4]. For example, primary frequency response from (utilityscale) DERs can significantly reduce the frequency nadir and steady-state frequency deviation [5]. Aligned with this emerging vision, this paper considers a distribution system featuring DERs, and proposes a DER-control strategy that enables a distribution feeder to emulate a virtual power plant

S. S. Guggilam and S. V. Dhople are with the Department of Electrical and Computer Engineering, University of Minnesota, Minneapolis, MN 55455, USA. E-mail: \{guggi022, sdhople\} @umn.edu. C. Zhao and E. Dall'Anese are with the National Renewable Energy Laboratory, Golden, CO. E-mail: \{changhong.zhao, emiliano.dallanese\}@nrel.gov. Y. C. Chen is with the Department of Electrical and Computer Engineering, The University of British Columbia, Vancouver, British Columbia V6T 1 Z4. E-mail: chen@ece.ubc.ca

This work was supported by the U.S. Department of Energy under Contract No. DE-AC36-08GO28308 with the National Renewable Energy Laboratory. The effort of S. Guggilam, C. Zhao, E. Dall'Anese, and S. V. Dhople was primarily supported by the Advanced Research Projects Agency-Energy (ARPA-E) under the Network Optimized Distributed Energy Systems (NODES) program. The U.S. Government retains and the publisher, by accepting the article for publication, acknowledges that the U.S. Government retains a nonexclusive, paid-up, irrevocable, worldwide license to publish or reproduce the published form of this work, or allow others to do so, for U.S. Government purposes.

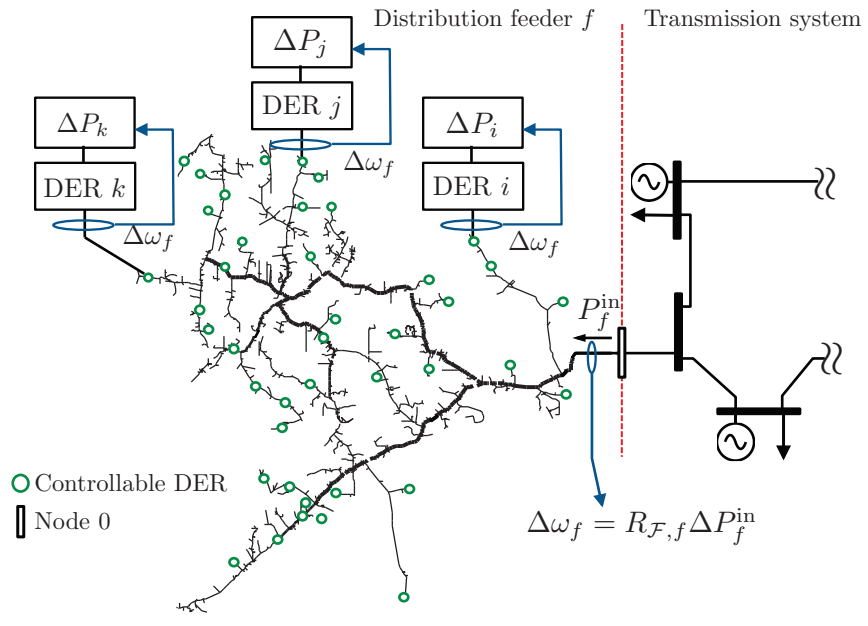

Fig. 1. The proposed approach demonstrates how power-frequency droop slopes for individual DERs $\left(m_{k}\right)$ can be designed so that: i) in aggregate, the $f$ distribution feeder presents a frequency-regulation characteristic, $R_{\mathcal{F}, f}$, at the feeder head, and ii) the injections of individual DERs $\left(\Delta P_{k}\right)$ are location agnostic, hence conforming to a well-defined notion of fairness.

effectively implementing a power-frequency-droop characteristic at the interface with the transmission grid.

We discuss ideas with respect to the illustrative system in Fig. 1. The objective is to design individual controllers for DERs located throughout a feeder $f$, so that the active power injected at the feeder head, $P_{f}^{\text {in }}$, is collectively adjusted in response to frequency deviations $\Delta \omega_{f}$ via the powerfrequency droop relationship $\Delta \omega_{f}=R_{\mathcal{F}, f} \Delta P_{f}^{\text {in }}$, where $R_{\mathcal{F}, f}$ is a given regulation constant. This approach enables a system-theoretic stability analysis at the transmission level, where distribution-level DER aggregations can be effectively modeled as virtual power plants that provide prescribed primary frequency response characteristics.

Targeting the goal outlined above, we develop a systematic procedure to design power-frequency droop characteristics by leveraging suitable linear approximations of the AC power-flow equations [6], [7]. The resultant droop coefficients intrinsically embed information regarding the locations of the DERs and, hence, the effect of each DER power adjustment $\Delta P_{k}$ on the feeder-level response $\Delta P_{f}^{\text {in }}$ [cf. Figure 1]. The proposed methodology also accommodates DER "participation factors," which can be designed either to ensure fairness across the DER power adjustments, or, based on (albeit futuristic) distribution-level frequencysupport markets. Overall, the resultant droop coefficients resemble traditional participation coefficients of large-scale generators in secondary frequency control settings in the 
bulk transmission system [8], [9]; in our case, these factors model the contribution of each DER towards achieving a prescribed (collective) regulation constant $R_{\mathcal{F}, f}$ at the feeder head, and account for both distribution-network power flows and fairness/market principles.

Prior work in this context have considered primary frequency control schemes for large-scale photovoltaic (PV) and wind plants [1], [5], [10]. At the distribution level, decentralized primary frequency control schemes have been explored leveraging PV systems [11], energy storage systems [4], [12], [13], or based on switching on/off HVAC systems, refrigerators, and water heaters when the magnitude and duration of local frequency deviation and the temperature hit certain thresholds [2], [3]. Small-scale experiments on residential appliances participating in frequency control have also been performed [14]-[16]. Overall, the effects of the DER location (and, hence, power flows within the feeder), operational constraints, and participation factors on the design and performance analysis of primary frequency control are not considered. Furthermore, ad-hoc and networkagnostic designs may not enable the distribution system to achieve a prescribed regulation constant $R_{\mathcal{F}, f}$ at the feeder head. A simulation study has demonstrated effectiveness of load-side participation in primary frequency control [17]. To the best of our knowledge, this paper is the first to propose a primary frequency control law for DERs that achieves a target aggregate performance, while simultaneously ensuring that the response of individual DERs is location agnostic.

The remainder of this paper is organized as follows. In Section II, the mathematical notation, network model, and dynamical models of generators and DERs are described. By analyzing the steady-state frequency, Section III presents the proposed method to engineer DER droop slopes while acknowledging the distribution feeder network. In Section IV, we present several numerical case studies to illustrate the proposed design method. Finally, concluding remarks and some directions for future work are highlighted in Section V.

\section{Preliminaries And System Model}

In this section, we first establish notation and then describe the power-system model.

\section{A. Notation}

Let $\mathbb{R}$ be the set of real numbers and $\mathbb{C}$ the set of complex numbers. Given a finite set $\mathcal{S} \subset \mathbb{R}$ we use $S=|\mathcal{S}|$ to denote its cardinality. The matrix inverse is denoted by $(\cdot)^{-1}$, transpose by $(\cdot)^{\mathrm{T}}$, complex conjugate by $(\cdot)^{*}$, real and imaginary parts of a complex number by $\operatorname{Re}\{\cdot\}$ and $\operatorname{Im}\{\cdot\}$, respectively, and $\mathrm{j}:=\sqrt{-1}$. The magnitude of a complex scalar is denoted by $|\cdot|$. A diagonal matrix formed with diagonal entries composed of entries of vector $x$ is denoted by $\operatorname{diag}(x)$. For a matrix $X, X_{m n}$ returns the entry in the

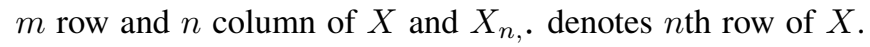
The $N \times 1$ vectors with all ones and all zeros are denoted by $1_{N}$ and $0_{N}$, respectively. The spaces of $N \times 1$ real-valued and complex-valued vectors are denoted by $\mathbb{R}^{N}$ and $\mathbb{C}^{N}$, respectively.

\section{B. Network Model}

In this section we introduce the notation used to describe the transmission and distribution systems.

1) Transmission Network: We consider a classical power network model for the transmission grid, which is represented by a graph, where $\mathcal{N}:=\{1, \ldots,|\mathcal{N}|\}$ is the set of buses, and $\mathcal{E} \subset \mathcal{N} \times \mathcal{N}$ is the set of transmission lines. A transmission line is denoted by $(g, \ell) \in \mathcal{E}$. Partition the set $\mathcal{N}=\mathcal{G} \cup \mathcal{L}$, where $\mathcal{G}$ is the set of buses that are connected to conventional turbine-based generators (high inertia) and $\mathcal{L}$ is the set of buses that are connected to loads (which can be aggregate representations of distribution feeders). The set of neighbors of bus $g$ is defined as $\mathcal{N}_{g}:=\{\ell \in \mathcal{N} \mid(g, \ell) \in \mathcal{E}\}$.

2) Distribution Network: Denote, by $\mathcal{F} \subset \mathcal{L}$, the set of buses in the transmission system where distribution feeders are present. Node 0 denotes the secondary side of the distribution transformer. Accordingly, nodes of the distribution feeder connected to transmission system bus $f \in \mathcal{F}$, comprising $B_{f}+1$ nodes, are collected in the set $\mathcal{B}_{f} \cup\{0\}$, $\mathcal{B}_{f}:=\left\{1, \ldots, B_{f}\right\}$, and lines represented by the set of edges $\left.\mathcal{E}_{f}:=\{(m, n)\} \subset\left(\mathcal{B}_{f} \cup\{0\}\right) \times\left(\mathcal{B}_{f} \cup\{0\}\right)\right\}$. Furthermore, for the distribution feeder connected to transmission system bus $f \in \mathcal{F}$, let $\mathcal{D}_{f} \subseteq \mathcal{B}_{f}$ with cardinality $D_{f}=\left|\mathcal{D}_{f}\right|$ denote the set of buses where controllable DERs are present. Let $V_{n} \in \mathbb{C}$ and $I_{n} \in \mathbb{C}$ denote the phasors for the lineto-ground voltage at and the current injected into node $n$, respectively, and define the $B_{f}$-dimensional complex vectors $v:=\left[V_{1}, \ldots, V_{B_{f}}\right]^{\mathrm{T}} \in \mathbb{C}^{B_{f}}$ and $i:=\left[I_{1}, \ldots, I_{B_{f}}\right]^{\mathrm{T}} \in \mathbb{C}^{B_{f}}$. Also, let $V_{0}$ denote the voltage at the secondary side of the distribution transformer, and let $I_{0}$ denote the current injected into the distribution feeder from the transmission system bus $f$. Using Ohm's and Kirchhoff's circuit laws, the following linear relationship can be established:

$$
\left[\begin{array}{c}
I_{0} \\
i
\end{array}\right]=\left[\begin{array}{cc}
y_{00} & \bar{y}^{\mathrm{T}} \\
\bar{y} & Y
\end{array}\right]\left[\begin{array}{l}
V_{0} \\
v_{g}
\end{array}\right]=: Y_{\text {net }}\left[\begin{array}{c}
V_{0} \\
v
\end{array}\right]
$$

where the system admittance matrix $Y_{\text {net }} \in \mathbb{C}^{\left(B_{f}+1\right) \times\left(B_{f}+1\right)}$ is formed based on the system topology and the $\pi$-equivalent circuit of the distribution lines (see e.g., [18, Chapter 6] for additional details on distribution line modeling), and is partitioned in sub-matrices with the following dimensions: $Y \in \mathbb{C}^{B_{f} \times B_{f}}, \bar{y} \in \mathbb{C}^{B_{f} \times 1}$, and $y_{00} \in \mathbb{C}$.

\section{Power-flow Equations}

Transmission line $(k, j)$ is modeled using the lumpedelement $\pi$-model with series admittance $y_{k j}=y_{j k}=g_{k j}+$ $\mathrm{j} b_{k j} \in \mathbb{C} \backslash\{0\}$ and shunt admittance $y_{k j}^{\mathrm{sh}}=g_{k j}^{\mathrm{sh}}+\mathrm{j} b_{k j}^{\mathrm{sh}} \in$ $\mathbb{C} \backslash\{0\}$ on both ends of the line. The power injections at buses $k \in \mathcal{N} \cup\left(\cup_{f \in \mathcal{F}} \mathcal{B}_{f}\right)$ are given by

$$
0=P_{k}-m_{k} \dot{\theta}_{k}-\sum_{j \in \mathcal{N}_{k}} P_{k j}, \quad 0=Q_{k}-\sum_{j \in \mathcal{N}_{k}} Q_{k j}
$$

where $P_{k}$ and $Q_{k}$ are the net non-frequency-sensitive activeand reactive-power injections at node $k$; and following standard power-flow computations, the branch flows $P_{k j}, Q_{k j}$, 
are given by

$$
\begin{aligned}
P_{k j}=\mid & \left.V_{k}\right|^{2}\left(g_{k j}^{\mathrm{sh}}+g_{k j}\right) \\
& -\left|V_{k}\right|\left|V_{j}\right|\left(g_{k j} \cos \theta_{k j}+b_{k j} \sin \theta_{k j}\right) \\
Q_{k j}=- & \left|V_{k}\right|^{2}\left(b_{k j}^{\mathrm{sh}}+b_{k j}\right) \\
& \quad-\left|V_{k}\right|\left|V_{j}\right|\left(g_{k j} \sin \theta_{k j}-b_{k j} \cos \theta_{k j}\right) .
\end{aligned}
$$

In (3), at bus $k$, frequency-sensitive active-power injections are $-m_{k} \dot{\theta}_{k}$ if bus $k$ is connected to DERs with droop slope $m_{k}$. We comment on this further in Section II-D.2 below.

\section{System Dynamical Models}

We describe the dynamical model for the generators in the transmission network, and then discuss the frequencyresponsive DER model.

1) Transmission Network: Since we are interested in time scales in the regime of primary frequency response, we model the dynamics of angular position, frequency, and mechanical-power input for the generators in the network. In particular, for the $g \in \mathcal{G}$ generator, we adopt the following third-order dynamical model:

$$
\begin{aligned}
\dot{\theta}_{g} & =\omega_{g}-\omega_{\mathrm{s}} \\
M_{g} \dot{\omega}_{g} & =P_{g}^{\mathrm{m}}-D_{g}\left(\omega_{g}-\omega_{\mathrm{s}}\right)+P_{g}-\sum_{k \in \mathcal{N}_{g}} P_{g k} \\
\tau_{g} \dot{P}_{g}^{\mathrm{m}} & =-P_{g}^{\mathrm{m}}+P_{g}^{\mathrm{r}}-\frac{1}{R_{\mathcal{G}, g}}\left(\omega_{g}-\omega_{\mathrm{s}}\right) .
\end{aligned}
$$

Above, $\theta_{g}, \omega_{g}$, and $P_{g}^{\mathrm{m}}$ are the dynamical states for rotor electrical angular position, generator frequency, and turbine mechanical power, respectively, for the $g$ generator, and $\omega_{\mathrm{s}}=$ $2 \pi 60\left[\mathrm{rad} \mathrm{sec}^{-1}\right]$ is the synchronous frequency. Furthermore, $M_{g}$ is the inertia constant, $D_{g}$ is the load damping coefficient (capturing the frequency-sensitive loads at bus $g$ ), $R_{\mathcal{G}, g}$ is the frequency-power speed-droop regulation constant, $\tau_{g}$ is the turbine time constant, and $P_{g}^{\mathrm{r}}$ denotes its reference power setting (assumed to be constant since it derives from secondary control). Finally, $P_{g}$ is the injection at bus $g$ (negative, if we wish to model a constant power load).

2) Distribution Network: Assume the following model for the power-electronics-based zero-inertia DERs connected to buses $k \in \mathcal{B}_{f}$ in feeder $f \in \mathcal{F}$

$$
\begin{aligned}
\dot{\theta}_{k} & =\omega_{f}-\omega_{\mathrm{s}} \\
0 & =P_{k}-\sum_{j \in \mathcal{N}_{k}} P_{k j}-m_{k} \dot{\theta}_{k} .
\end{aligned}
$$

The above model is appropriate for DERs in a setting where the frequency at the feeder head (connected to the transmission network) percolates down to all buses in the feeder [17]. The droop coefficient $m_{k}$ establishes the frequency response of the DER at bus $k$. Recall that we define $\mathcal{D}_{f} \subseteq \mathcal{B}_{f}$ to be the subset of nodes with frequency-responsive DERs and note that $m_{k}>0, \forall k \in \mathcal{D}_{f}$; and $m_{k}=0, \forall k \in \mathcal{B}_{f} \backslash \mathcal{D}_{f}$. For simplicity and ease of subsequent exposition, we assume that the power rating of each DER is identical for each feeder. Extensions to the case where DER capacities are significantly different are beyond the scope of the present work, and they remain the focus of ongoing investigations.

\section{FREQUENCY RESPONSE OF TRANSMISSION AND DISTRIBUTION NETWORK}

In this section, we quantify the steady-state frequency offset that results from a power imbalance in the network. Subsequently, we describe how the DER droop slopes can be engineered to yield a desired frequency regulation for the combined transmission-distribution network while acknowledging power flows in the distribution feeder.

\section{A. System Steady-state Frequency}

Assume the system initially operates at the synchronous steady-state equilibrium point with $\omega_{g}^{\circ}=\omega_{\mathrm{s}}$. Accordingly, from (4), at the initial steady state, for generator $g \in \mathcal{G}$,

$$
\begin{aligned}
& 0=P_{g}^{\mathrm{m} \circ}+P_{g}^{\circ}-\sum_{k \in \mathcal{N}_{g}} P_{g k}^{\circ} \\
& 0=-P_{g}^{\mathrm{m} \circ}+P_{g}^{\mathrm{r}} .
\end{aligned}
$$

Then, suppose an imbalance in active-power generation and consumption arises in the transmission network. The system would eventually settle to a new steady state. Since we are interested in a time horizon where secondary control has not yet acted (i.e., $P_{g}^{\mathrm{r}}$ remains constant), this steady state does not correspond to the nominal (synchronous) frequency. Define new steady-state quantities $\widetilde{\omega}_{g}=\omega_{\mathrm{s}}+\Delta \omega_{g}, \widetilde{P}_{g}^{\mathrm{m}}=$ $P_{g}^{\mathrm{mo}}+\Delta P_{g}^{\mathrm{m}}, \widetilde{P}_{g}=P_{g}^{\circ}+\Delta P_{g}, \widetilde{P}_{g k}=P_{g k}^{\circ}+\Delta P_{g k}, \forall g \in$ $\mathcal{G}, k \in \mathcal{N}_{g}$. With reference to the model in (4), at the new steady state, for generator $g \in \mathcal{G}$,

$$
\begin{aligned}
& 0=\widetilde{P}_{g}^{\mathrm{m}}-D_{g}\left(\widetilde{\omega}_{g}-\omega_{\mathrm{s}}\right)+\widetilde{P}_{g}-\sum_{k \in \mathcal{N}_{g}} \widetilde{P}_{g k} \\
& 0=-\widetilde{P}_{g}^{\mathrm{m}}+P_{g}^{\mathrm{r}}-\frac{1}{R_{\mathcal{G}, g}}\left(\widetilde{\omega}_{g}-\omega_{\mathrm{s}}\right) .
\end{aligned}
$$

Substituting the new steady-state variables defined above and recognizing (6), we get from (7) that

$$
\begin{aligned}
0 & =\Delta P_{g}^{\mathrm{m}}-D_{g} \Delta \omega_{g}+\Delta P_{g}-\sum_{k \in \mathcal{N}_{g}} \Delta P_{g k} \\
\Delta P_{g}^{\mathrm{m}} & =-\frac{1}{R_{\mathcal{G}, g}} \Delta \omega_{g} .
\end{aligned}
$$

Finally, substitute (9) into (8) to get, for each $g \in \mathcal{G}$,

$$
\left(R_{\mathcal{G}, g}^{-1}+D_{g}\right) \Delta \omega_{g}=\Delta P_{g}-\sum_{k \in \mathcal{N}_{g}} \Delta P_{g k}=: \Delta P_{\text {dist }}^{g} .
$$

Similarly, define new steady-state quantities $\widetilde{\omega}_{f}=\omega_{\mathrm{s}}+$ $\Delta \omega_{f}, \forall f \in \mathcal{F} ; \widetilde{P}_{k}=P_{k}^{\circ}+\Delta P_{k}$ and $\widetilde{P}_{k j}=P_{k j}^{\circ}+\Delta P_{k j}$, $\forall k \in \mathcal{B}_{f}$ and $j \in \mathcal{N}_{k}$. With reference to the model for the DERs in (5), at the new steady state, for the DER at bus $k \in \mathcal{B}_{f}$,

$$
0=\widetilde{P}_{k}-\sum_{j \in \mathcal{N}_{k}} \widetilde{P}_{k j}-m_{k}\left(\widetilde{\omega}_{f}-\omega_{\mathrm{s}}\right) .
$$

Substituting the new steady-state quantities defined above and recognizing that, initially, $\omega_{f}^{\circ}=\omega_{\mathrm{s}}$, we get that for a particular distribution feeder $f \in \mathcal{F}$, and for all $k \in \mathcal{B}_{f}$,

$$
m_{k} \Delta \omega_{f}=\Delta P_{k}-\sum_{j \in \mathcal{N}_{k}} \Delta P_{k j} .
$$


Summing (12) over all $k \in \mathcal{B}_{f}$, we get

$$
\sum_{k \in \mathcal{B}_{f}} m_{k} \Delta \omega_{f}=\sum_{k \in \mathcal{B}_{f}}\left(\Delta P_{k}-\sum_{j \in \mathcal{N}_{k}} \Delta P_{k j}\right)=: \Delta P_{\text {dist }}^{f} .
$$

At the post-disturbance steady state, the power flows in the network settle to a new equilibrium, and so for any pair of buses $k, j \in \mathcal{G} \cup \mathcal{F}, \theta_{k j}=\theta_{k}-\theta_{j}$ is a constant. Consequently, we have that $\forall k, j \in \mathcal{G} \cup \mathcal{F}, \dot{\theta}_{k j}=\dot{\theta}_{k}-\dot{\theta}_{j}=\Delta \omega_{k}-\Delta \omega_{j}=0$. Thus, at the new steady state, we get that

$$
\Delta \omega_{k}=: \Delta \omega^{\star}, \forall k \in \mathcal{G} \cup \mathcal{F}
$$

where $\Delta \omega^{\star}$ denotes the system-wide steady-state frequency offset. With this in mind, summing equalities in (10) over all generators $g \in \mathcal{G}$ and (13) over all feeders $f \in \mathcal{F}$, we get

$$
\begin{aligned}
& \left(\sum_{g \in \mathcal{G}}\left(R_{\mathcal{G}, g}^{-1}+D_{g}\right)+\sum_{f \in \mathcal{F}} \sum_{k \in \mathcal{B}_{f}} m_{k}\right) \Delta \omega^{\star} \\
& =\left(\sum_{g \in \mathcal{G}}\left(R_{\mathcal{G}, g}^{-1}+D_{g}\right)+\sum_{f \in \mathcal{F}} \sum_{k \in \mathcal{D}_{f}} m_{k}\right) \Delta \omega^{\star} \\
& =\sum_{g \in \mathcal{G}} \Delta P_{\text {dist }}^{g}+\sum_{f \in \mathcal{F}} \Delta P_{\text {dist }}^{f}=: \Delta P_{\text {dist }}
\end{aligned}
$$

where the second equality follows from the fact that $m_{k}=$ $0, \forall k \in \mathcal{B}_{f} \backslash \mathcal{D}_{f}$. Rearranging terms in (15), and isolating $\Delta \omega^{\star}$, we get that

$$
\Delta \omega^{\star}=\frac{\Delta P_{\text {dist }}}{\sum_{g \in \mathcal{G}}\left(R_{\mathcal{G}, g}^{-1}+D_{g}\right)+\sum_{f \in \mathcal{F}} \sum_{k \in \mathcal{D}_{f}} m_{k}}=: \frac{\Delta P_{\text {dist }}}{R_{\text {eff }}^{-1}}
$$

where $R_{\text {eff }}^{-1}$ is the network-wide frequency regulation characteristic, and it dictates the expected frequency offset given a change in power balance from the pre-disturbance frequencysynchronized equilibrium.

\section{B. A Notion of Fairness}

We will find it useful to disaggregate the frequency regulation expected from all feeders into a sum of expected contributions from each feeder by defining the frequency regulation constant for the $f$ feeder as

$$
R_{\mathcal{F}, f}^{-1}:=\sum_{k \in \mathcal{D}_{f}} m_{k}
$$

With this definition in place, it follows straightforwardly that

$$
R_{\text {eff }}=\sum_{g \in \mathcal{G}}\left(R_{\mathcal{G}, g}^{-1}+D_{g}\right)+\sum_{f \in \mathcal{F}} R_{\mathcal{F}, f}^{-1} .
$$

Indeed, there are infinite options to pick the individual $m_{k}$ 's such that (17) holds. Under the presumed modeling constraint of the DERs having the same nominal power rating, but we focus on a particular choice that guarantees fairness of response. By this we mean that the change in active-power outputs of DERs in feeder $f \in \mathcal{F}$ are such that

$$
\Delta P_{1}=\Delta P_{2}=\cdots=\Delta P_{D_{f}} .
$$

With the definition of the per-feeder frequency-regulation characteristic in (17), the design problem is the following:
How does one pick the power-frequency droop slopes for individual DERs $\left(m_{k}\right)$ so that: $\left.i\right)$ in aggregate, the distribution feeder presents a frequency-regulation characteristic, $R_{\mathcal{F}, f}$, at the feeder head, and ii) the injections of individual DERs $\left(\Delta P_{k}\right)$ can be engineered to conform to the fairness objective in (19)?

At first glance, the solution to the above design problem is not obvious, given the topological and compositional complexity of distribution networks. However, we demonstrate next how a linearization of the nonlinear power-flow equations for each feeder can aid in this design.

\section{Designing the Droop Coefficients}

For a particular feeder $f \in \mathcal{F}$, we would like to achieve

$$
\Delta P_{f}^{\text {in }}=R_{\mathcal{F}, f}^{-1}\left(\omega_{f}-\omega_{\mathrm{s}}\right)
$$

where $\Delta P_{f}^{\mathrm{in}}$ is the change in power flowing in from the feeder head into the distribution network. (See Fig. 1 for an illustration.) The key idea is to disaggregate the change in injection at the feeder head into individual contributions from injection changes at different nodes in the feeder. Following the developments in Appendix A, we reproduce (43) below:

$$
\Delta P_{f}^{\mathrm{in}}=\sum_{k \in \mathcal{B}_{f}} \varphi_{k} \Delta P_{k}
$$

where $\Delta P_{k}$ is the change in injection at the DER installed at bus $k$ in the feeder, and $\varphi_{k}$ takes the connotation of a participation factor, that quantifies how much a change in injection at a given node in the feeder contributes to the total change in the flow at the feeder head. The approach outlined in Appendix A leverages a straightforward linearization of the power-flow equations on a per-feeder basis to compute $\varphi_{k}, \forall k \in \mathcal{B}_{f}$. The values of $\varphi_{k}$ are different from -1 in realistic settings (due to lossy lines, non-negligible shunt loads, or, in the trivial case, the absence of frequencysensitive injections). We also note that the magnitudes of $\varphi_{k}$ tend to decrease for nodes that are farther away from the feeder head. This makes intuitive sense, since it implies that incremental changes in injections significantly downstream from the feeder head tend to have less impact on the injection change at the feeder head. ${ }^{1}$ With these introductory remarks in place, we substitute (21) in (20), to obtain

$$
\omega_{f}-\omega_{\mathrm{s}}=R_{\mathcal{F}, f} \sum_{k \in \mathcal{B}_{f}} \varphi_{k} \Delta P_{k}
$$

The $k$ DER is then designed to respond as follows

$$
\Delta P_{k}=\frac{\gamma_{k}}{R_{\mathcal{F}, f} \varphi_{k}}\left(\omega_{f}-\omega_{\mathrm{s}}\right)=\frac{\gamma_{k}}{R_{\mathcal{F}, f} \varphi_{k}} \dot{\theta}_{k} .
$$

Above, $\gamma_{k}>0, \forall k \in \mathcal{D}_{f}$, where $\mathcal{D}_{f} \subseteq \mathcal{B}_{f}$ is the subset of nodes with frequency-responsive DERs; $\gamma_{k}=0, \forall k \in$ $\mathcal{B}_{f} \backslash \mathcal{D}_{f}$. Furthermore, the $\gamma_{k}$ values have to be picked such that $\sum_{k \in \mathcal{D}_{f}} \gamma_{k}=1$.

\footnotetext{
${ }^{1}$ Demonstrating this analytically is beyond the scope of the work, but we provide numerical results subsequently to substantiate this claim.
} 


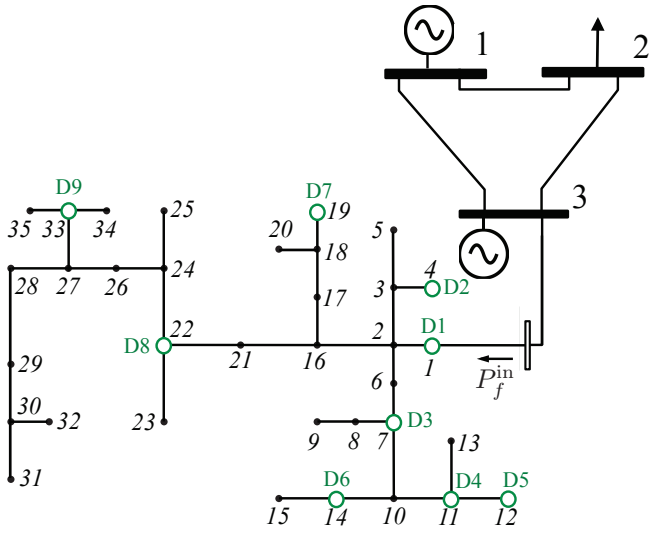

Fig. 2. Power network used in the case study includes a transmission network with 3 buses, and a 36-node distribution network connected to bus 2 in the transmission system. The distribution feeder includes nine frequencyresponsive DERs, D1, ., D9.

While all choices of $\gamma_{k}$ as described above yield the required frequency-regulation characteristic for each feeder, different choices lead to different participatory models for the DERs. For instance, a straightforward (albeit naïve) option would be to choose

$$
\gamma_{k}=D_{f}^{-1}=:\left|\mathcal{D}_{f}\right|^{-1}
$$

which conforms to the requirement that $\sum_{\ell \in \mathcal{D}_{f}} \gamma_{\ell}=1$. With this choice, we get

$$
\Delta P_{k}=\frac{D_{f}^{-1}}{R_{\mathcal{F}, f} \varphi_{k}}\left(\omega_{f}-\omega_{\mathrm{s}}\right) .
$$

As noted earlier, since the (magnitude of) $\varphi_{k}$ tends to reduce as we move downstream from the feeder head, with the model in (25), DERs that are electrically distant from the feeder head contribute-admittedly unfairly_more while providing primary-frequency response.

Alternatively, the design strategy that ensures fairness of participation, i.e., $\Delta P_{1}=\cdots=\Delta P_{D_{f}}$, is to pick

$$
\gamma_{k}=\frac{\varphi_{k}}{\sum_{\ell \in \mathcal{D}_{f}} \varphi_{\ell}}
$$

Indeed, with this choice, it follows from (23) that

$$
\Delta P_{1}=\cdots=\Delta P_{D_{f}}=\frac{1}{R_{\mathcal{F}, f} \sum_{\ell \in \mathcal{D}_{f}} \varphi_{\ell}}\left(\omega_{f}-\omega_{\mathrm{s}}\right) .
$$

In this case, all DERs provide the same change in injection, and they are not penalized for their location on the feeder.

We conclude by remarking that other choices of $\gamma_{k}$ would translate to alternative notions of fairness. Due to space constraints, we restrict the scope of the present paper and the subsequent numerical case studies to focus on the notion of fairness discussed in (19).

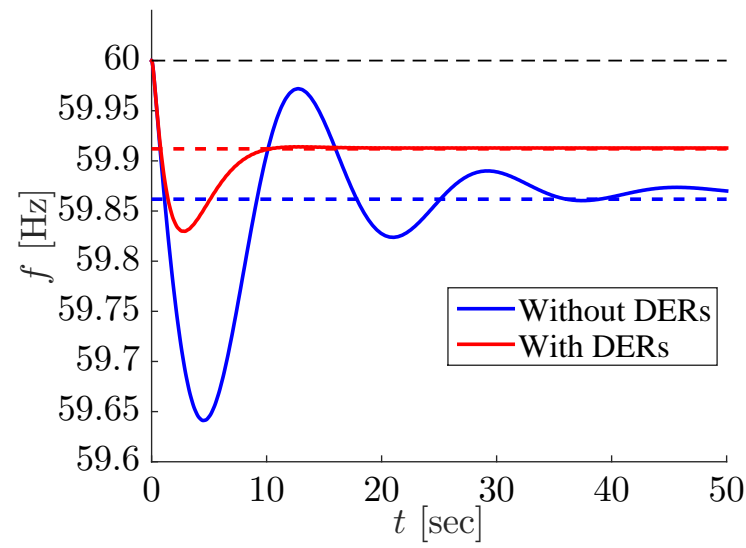

Fig. 3. Frequency at bus 3 after the load step at bus 2. The transient and steady-state response with frequency-responsive DERs is significantly improved. Steady-state results from time-domain simulations (solid lines) match the analytical results (dashed lines).

\section{CASE STUdiES}

We simulate a 3-bus transmission system, $\mathcal{N}=\{1,2,3\}$, with a generator connected at buses $\mathcal{G}=\{1,3\}$ and a 36-bus distribution feeder with frequency-responsive DERs at $\mathcal{F}=\{3\}$, as shown in Fig. 2. The distribution feeder contains frequency-responsive DERs, D1, ., D9, at nodes $\mathcal{D}_{3}=\{2,5,8,12,13,15,20,23,34\}$. The 3-bus transmission system is a modified version of a test case in [19]. The 36-bus distribution feeder, whose head bus is connected to transmission bus 3 , is a suitably modified version of the IEEE 37-bus distribution test feeder [20]. The network parameters of the 3-bus system, and power-flow states corresponding to the pre-disturbance steady state are listed in Appendix B. For convenience, voltage magnitudes are in per unit $(\mathrm{pu})$ with a $4.8[\mathrm{kV}]$ base, and power and impedance values are also in (pu), unless otherwise specified. Ensuing results are obtained from time-domain simulations of the combined transmissiondistribution-network model from Section II, performed with the Power System Toolbox [21].

At time $t=0[\mathrm{sec}]$, the load at bus 2 in the transmission network undergoes a step increase of 0.00217 . In our case studies, we consider four possible scenarios in which primary frequency response is provided due to the load change, described as follows:

(A) Only the two generators in the transmission network provide primary frequency response. None of the DERs are frequency responsive, i.e., $m_{\ell}=0, \forall \ell \in \mathcal{D}_{3}$.

(B) In addition to the generators, 9 DERs across the 36bus distribution feeder collected in the set $\mathcal{D}_{3}$ also contribute to primary frequency response. Here, the prescribed frequency regulation constant at the feeder head is $R_{\mathcal{F}, 3}^{-1}=0.5208$. Droop slopes for individual DERs are naïvely selected so that their outputs are given by (25).

(C) The DERs continue to provide frequency support. Here, we consider a set of arbitrary droop slopes that only conform to the requirement $R_{\mathcal{F}, 3}^{-1}=0.5208$. 
(a)

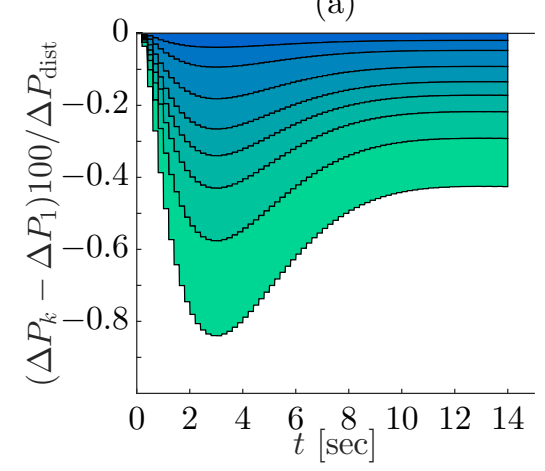

(b)

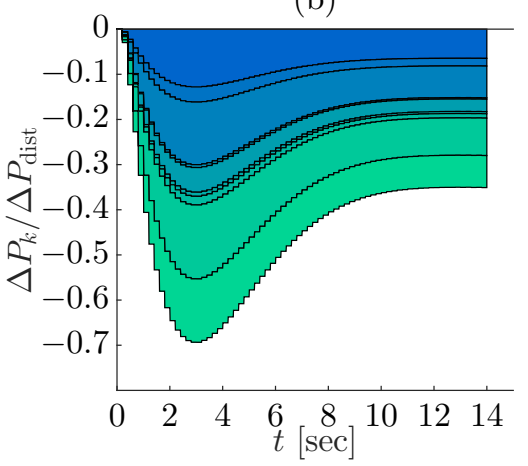

(c)

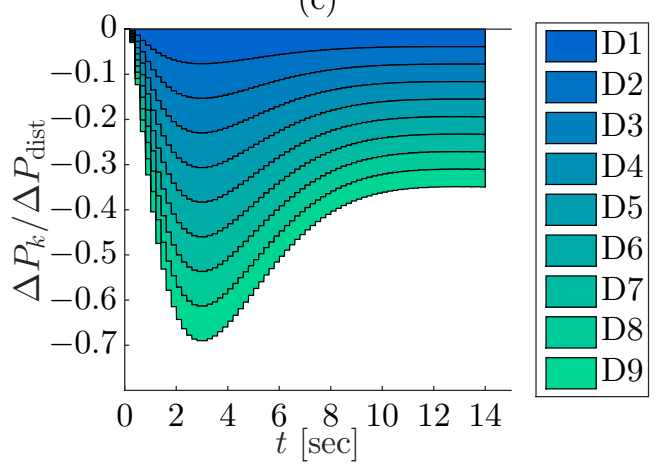

Fig. 4. (a) Relative contributions of each DER (with respect to the one installed closest to the feeder head) indicate how a naïve choice of droop coefficients penalizes the ones that are far from the feeder head. (b) An arbitrary choice of droop coefficients results in arbitrary contributions from the DERs. (c) Droop slopes are engineered such that DERs provide the same injections irrespective of their location on the feeder.

(D) Finally, with the same prescribed aggregate droop $R_{\mathcal{F}, 3}^{-1}=0.5208$, and to promote a notion of fairness, the droop coefficients for individual DERs are designed such that their outputs are given by (27).

\section{A. Transient Performance and Steady-state Frequency}

Figure 3 shows the time evolution of frequency at bus 3 in the transmission network for the cases without (i.e., case (A)) and with (i.e., cases (B), (C), (D)) DER frequency support. In case (A), where only the generators provide frequency support, from (16), we expect that

$$
\Delta \omega^{\star}=\frac{\Delta P_{\text {dist }}}{\sum_{g \in \mathcal{G}}\left(D_{g}+R_{\mathcal{G}, g}^{-1}\right)}=0.002304
$$

which corresponds to a steady-state frequency of 59.86 [Hz]. This is confirmed by the simulation result in Fig. 3. In cases (B), (C), and (D), where the DERs are also engaged in frequency response, we expect from (16) that

$$
\Delta \omega^{\star}=\frac{\Delta P_{\text {dist }}}{\sum_{g \in \mathcal{G}}\left(D_{g}+R_{\mathcal{G}, g}^{-1}\right)+\sum_{\ell \in \mathcal{D}_{3}} m_{\ell}}=0.00149
$$

which corresponds to a steady-state frequency of $59.91[\mathrm{~Hz}]$, also confirmed by simulation results shown in Fig. 3. Since the 9 DERs are coordinated to provide the same frequencyregulation constant at the feeder head, in all three cases, the transient response and steady-state behaviour are the same.

Broadly speaking, the results shown in Fig. 3 confirm that controlling the DERs leads to a better transient performance, with faster convergence and smaller oscillations, than the case where only the generators provide frequency response. The main reason is that the DERs instantly respond to frequency deviations unlike the generators. Furthermore, the match in steady-state between the time-domain simulation results (in solid lines) and the analytical results (dashed lines) validates the analytical developments in Section III.

\section{B. Comparison of Cases $(B),(C)$, and $(D)$}

By selecting droop coefficients as in (25), i.e., Case (B), we obtain the trend that DERs farther away from the feeder head contribute more active power while providing primary frequency support. This makes intuitive sense, since incremental changes in injections significantly downstream from the feeder head tend to have less impact on the injection change at the feeder head. In order to see this effect, we plot the normalized quantity $\left(\Delta P_{k}-\Delta P_{1}\right) 100 / \Delta P_{\text {dist }}$ in Fig. $4 \mathrm{a}$, where $\Delta P_{1}$ is the change in active-power injection at the DER closest to the feeder head.

With the arbitrary droop coefficients discussed in case (C), we plot the quantities $\Delta P_{k} / \Delta P_{\text {dist }}$ in Fig. 4b. In this setup, we see that the injections are arbitrary and they are not systematically engineered to conform to any fairness objective. In contrast, with the allocation defined in (27), i.e., case (D), we plot the quantities $\Delta P_{k} / \Delta P_{\text {dist }}$ in Fig. 4c. This clearly promotes a sense of fairness among the DERs since they all contribute equally to offer primary frequency response.

Notice from Fig. 4b and Fig. 4c that although individual DER contributions vary (based on the choice of the design coefficient $\gamma_{k}$ ), the total contribution of DERs (i.e. $\left.\sum_{k \in \mathcal{D}_{f}} \Delta P_{k}\right)$ remains the same.

\section{CONCluding Remarks AND ONGOING WORK}

In this paper, we proposed a method to design powerfrequency droop characteristics for individual DERs in a distribution feeder so that, in aggregate, they provide a prescribed primary frequency response at the feeder head. The method leverages linear approximations of the AC powerflow equations to embed participation factors into the design of the droop slopes such that the change in injections from the DERs can be engineered to be location agnostic. In the present work, DERs have been assumed to have the same nominal power rating, however, ongoing research includes extending this to consider DERs with different power ratings. Other avenues for future work include developing systematic methods for stability and convergence analyses, and real-time implementations of proposed algorithms. 


\section{APPENDIX}

\section{A. Approximation of Power Flowing into Distribution Feeder}

Let $s \in \mathbb{C}^{B_{f}}$ collect the complex-power injections at buses in $\mathcal{B}_{f}$ in feeder $f \in \mathcal{F}$. Then, using (1), the complex-power injections can be compactly written as

$$
s=\operatorname{diag}(v) i^{*}=\operatorname{diag}(v)\left(Y^{*} v^{*}+\bar{y}^{*} V_{0}^{*}\right) .
$$

Central to the linearization approach is to express the voltages $v$ as $v=v_{\text {nom }}+\Delta v$, where $v_{\text {nom }}$ is the linearization point, and entries of $\Delta v$ capture perturbations around $v_{\text {nom }}$. With $v_{\text {nom }}$ appropriately determined, we need to solve for $\Delta v$ that satisfies

$$
s=\operatorname{diag}\left(v_{\text {nom }}+\Delta v\right)\left(Y^{*}\left(v_{\text {nom }}+\Delta v\right)^{*}+\bar{y}^{*} V_{0}^{*}\right) .
$$

Neglecting second-order terms, we can show that $\Delta v$ satisfies

$$
\Gamma \Delta v+\Xi \Delta v^{*}=s-s_{\text {nom }}
$$

where $\Gamma, \Xi \in \mathbb{C}^{B_{f} \times B_{f}}$, and $s_{\text {nom }} \in \mathbb{C}^{B_{f}}$ are given by

$$
\begin{aligned}
\Gamma & :=\operatorname{diag}\left(Y^{*} v_{\text {nom }}^{*}+\bar{y}^{*} V_{0}^{*}\right) \\
\Xi & :=\operatorname{diag}\left(v_{\text {nom }}\right) Y^{*} \\
s_{\text {nom }} & :=\operatorname{diag}\left(v_{\text {nom }}\right)\left(Y^{*} v_{\text {nom }}^{*}+\bar{y}^{*} V_{0}^{*}\right) .
\end{aligned}
$$

Decomposing all quantities in (30) into their real and imaginary parts, we can solve for $\operatorname{Re}\{\Delta v\}$ and $\operatorname{Im}\{\Delta v\}$ (and hence, for $\Delta v$ ) from

$$
\left[\begin{array}{c}
\operatorname{Re}\{\Delta v\} \\
\operatorname{Im}\{\Delta v\}
\end{array}\right]=H\left[\begin{array}{c}
p \\
q
\end{array}\right]-H\left[\begin{array}{c}
p_{\text {nom }} \\
q_{\text {nom }}
\end{array}\right]
$$

where $s_{\text {nom }}=p_{\text {nom }}+\mathrm{j} q_{\text {nom }}$ and $s=p+\mathrm{j} q$ and we define $H \in \mathbb{R}^{2 B_{f} \times 2 B_{f}}$ as follows:

$$
H:=\left[\begin{array}{cc}
\operatorname{Re}\{\Gamma\}+\operatorname{Re}\{\Xi\} & -\operatorname{Im}\{\Gamma\}+\operatorname{Im}\{\Xi\} \\
\operatorname{Im}\{\Gamma\}+\operatorname{Im}\{\Xi\} & \operatorname{Re}\{\Gamma\}-\operatorname{Re}\{\Xi\}
\end{array}\right]^{-1}
$$

To aid subsequent discussions, we will find it useful to denote the $B_{f} \times B_{f}$ blocks that $H$ is composed of by: $H^{(11)}, H^{(12)}$, $H^{(21)}$, and $H^{(22)}$. This allows us to express

$$
\begin{aligned}
& \operatorname{Re}\{\Delta v\}=H^{(11)} p_{\text {inj }}+H^{(12)} q_{\text {inj }}+h_{r} \\
& \operatorname{Im}\{\Delta v\}=H^{(21)} p_{\text {inj }}+H^{(22)} q_{\text {inj }}+h_{i}
\end{aligned}
$$

where $h_{r}:=-H^{(11)} p_{\text {nom }}-H^{(12)} q_{\text {nom }}$ and $h_{i}:=$ $-H^{(21)} p_{\text {nom }}-H^{(22)} q_{\text {nom }}$.

Consider one particular test feeder $f \in \mathcal{F}$. Assume that node 1 is connected to the substation via a distribution line, and define the power entering the feeder by $S_{f}^{\text {in }}=V_{0} I_{01}^{*}$, where $I_{01}$ is the current flowing on the distribution line $(0,1)$. With $y_{01} \in \mathbb{C}$ denoting the admittance of line $(0,1), y_{00}^{\text {sh }} \in \mathbb{C}$ any passive shunt elements connected to node 0 , and $y_{01}^{\text {sh }}$ the shunt component of the line $(0,1)$, note that $I_{01}$ is given by $I_{01}=y_{01}\left(V_{0}-V_{1}\right)+y_{0} V_{0}$, with

$$
y_{0}=g_{0}+\mathrm{j} b_{0}:=y_{00}^{\mathrm{sh}}+y_{01}^{\mathrm{sh}} \text {. }
$$

Thus, $S_{f}^{\text {in }}$ can be rewritten as

$$
S_{f}^{\text {in }}=\left|V_{0}\right|^{2}\left(y_{01}^{*}+y_{0}^{*}\right)-V_{0}\left(y_{01}^{*} V_{1}^{*}\right) .
$$

With $\left\{e_{i} \in \mathbb{R}^{B_{f}}\right\}_{i=1}^{B_{f}}$ denoting the vector basis for $\mathbb{R}^{B_{f}}$, it follows that $V_{1}$ can be rewritten as

$$
V_{1}=e_{1}^{\mathrm{T}} v=e_{1}^{\mathrm{T}}\left(v_{\text {nom }}+\operatorname{Re}\{\Delta v\}+\operatorname{jIm}\{\Delta v\}\right)
$$

and, thus:

$$
S_{f}^{\text {in }}=\left|V_{0}\right|^{2}\left(y_{01}^{*}+y_{0}^{*}\right)-V_{0} y_{01}^{*}\left(e_{1}^{\mathrm{T}}\left(v_{\text {nom }}+\Delta v\right)\right)^{*} .
$$

Substituting (36) in (40) and rearranging terms, an approximate linear relationship between the power at the feeder head $S_{f}^{\text {in }}=P_{f}^{\text {in }}+\mathrm{j} Q_{f}^{\text {in }}$ and the net power injections $p, q$ throughout the feeder can be expressed as:

$$
\left[\begin{array}{c}
P_{f}^{\text {in }} \\
Q_{f}^{\text {in }}
\end{array}\right]=\left[\begin{array}{l}
\varphi \\
\zeta
\end{array}\right] p+\left[\begin{array}{c}
\alpha \\
\beta
\end{array}\right] q+c
$$

with $\varphi \in \mathbb{R}^{1 \times B_{f}}, \zeta \in \mathbb{R}^{1 \times B_{f}}, \alpha \in \mathbb{R}^{1 \times B_{f}}, \beta \in \mathbb{R}^{1 \times B_{f}}$, and $c \in \mathbb{R}^{2}$, each expressed as

$$
\begin{aligned}
& {\left[\begin{array}{c}
\varphi \\
\zeta \\
\alpha \\
\beta
\end{array}\right]=\left[\begin{array}{cccc}
-\psi_{1} & 0 & \psi_{2} & 0 \\
\psi_{2} & 0 & \psi_{1} & 0 \\
0 & -\psi_{1} & 0 & \psi_{2} \\
0 & \psi_{2} & 0 & \psi_{1}
\end{array}\right]\left[\begin{array}{c}
H_{1, \cdot}^{11} \\
H_{1, \cdot}^{12} \\
H_{1, \cdot}^{21} \\
H_{1, \cdot}^{22}
\end{array}\right]} \\
& c=\left|V_{0}\right|^{2}\left[\begin{array}{cccc}
1 & 1 & 0 & 0 \\
0 & 0 & -1 & -1
\end{array}\right]\left[g_{01}, g_{0}, b_{01}, b_{0}\right]^{\mathrm{T}} \\
& +\left[\begin{array}{cccc}
-\psi_{1} & \psi_{2} & -\psi_{1} & \psi_{2} \\
\psi_{2} & \psi_{1} & \psi_{2} & \psi_{1}
\end{array}\right]\left[\begin{array}{c}
\operatorname{Re}\left\{v_{\text {nom }, 1}\right\} \\
\operatorname{Im}\left\{v_{\text {nom }, 1}\right\} \\
-H_{1, \cdot}^{11} p_{\text {nom }}-H_{1, \cdot}^{12} q_{\text {nom }} \\
-H_{1, \cdot}^{21} p_{\text {nom }}-H_{1, \cdot}^{22} q_{\text {nom }}
\end{array}\right]
\end{aligned}
$$

and where the following scalars are defined for conciseness:

$$
\begin{aligned}
& \psi_{1}=\left|V_{0}\right|\left(\cos \left(\theta_{0}\right) g_{01}+\sin \left(\theta_{0}\right) b_{01}\right) \\
& \psi_{2}=\left|V_{0}\right|\left(\cos \left(\theta_{0}\right) b_{01}-\sin \left(\theta_{0}\right) g_{01}\right) .
\end{aligned}
$$

Now, consider that small perturbations in entries of $p$ result in the following model for the change in active-power flow at the feeder head:

$$
\Delta P_{f}^{\mathrm{in}} \approx \sum_{k \in \mathcal{B}_{f}} \varphi_{k} \Delta P_{k}
$$

which follows from (41) by neglecting entries of $\alpha$ (we observe empirically that they are small; implying that reactive power injections have minimal impact on the active-power flow on the feeder head), and recognizing that $c$ is a vector with constant entries.

\section{B. Simulation parameters}

The synchronous frequency, $\omega_{\mathrm{s}}=2 \pi 60\left[\mathrm{rad} \mathrm{sec}^{-1}\right]$. All values are reported in per unit unless otherwise noted.

Parameters of Transmission System: The generator damping coefficients are: $D_{1}=D_{3}=0.0434$, inertia constants are: $M_{1}=M_{3}=5$ [sec], droop coefficients are: $R_{\mathcal{G}, 1}^{-1}=$ 0.3472 and $R_{\mathcal{G}, 3}^{-1}=0.5208$, turbine time constants are $\tau_{1}=\tau_{3}=7$ [sec], reference power values are $P_{1}^{\mathrm{r}}=$ $0.01736, P_{3}^{\mathrm{r}}=0.02634, Q_{1}^{\mathrm{r}}=0.00512$ and $Q_{3}^{\mathrm{r}}=0.0161$. The load injections values are $P_{2}=-0.01558$ and $Q_{2}=$ -0.00651 . The transmission line parameters are given as $y_{12}=0.5+\mathrm{j} 5, y_{23}=0.5+\mathrm{j} 5$ and $y_{31}=1+\mathrm{j} 10$. The 
steady state values prior to disturbance for each transmission bus is given by, $V_{1}^{\circ}=1.01, V_{2}^{\circ}=0.984, V_{3}^{\circ}=1, \theta_{1}^{\circ}=$ $2.682[\mathrm{deg}], \theta_{2}^{\circ}=-0.817[\mathrm{deg}]$, and $\theta_{3}^{\circ}=0$ [deg].

Parameters of Distribution System: The network topology and power injections are in accordance to the standard IEEE 37-bus test feeder [20]. We add $y_{k k}^{\mathrm{sh}}=0.004+\mathrm{i} 0.005, \forall k \in$ $\{2, \ldots, 19\} \subset \mathcal{B}_{3}$ and $y_{k k}^{\text {sh }}=0.006+\mathrm{i} 0.007, \forall k \in$ $\{20, \ldots, 36\} \subset \mathcal{B}_{3}$. The base voltage is $4.8[\mathrm{kV}]$. The droop slope is $R_{\mathcal{F}, 3}^{-1}=0.5208$.

\section{REFERENCES}

[1] V. Gevorgian and B. O'Neill, "Advanced grid-friendly controls demonstration project for utility-scale PV power plants," National Renewable Energy Laboratory, Golden, CO, USA, Tech. Rep., 2016.

[2] J. A. Short, D. G. Infield, and L. L. Freris, "Stabilization of grid frequency through dynamic demand control," IEEE Transactions on Power Systems, vol. 22, no. 3, pp. 1284-1293, Jul 2007.

[3] A. Molina-Garcia, F. Bouffard, and D. S. Kirschen, "Decentralized demand-side contribution to primary frequency control," IEEE Transactions on Power Systems, vol. 26, no. 1, pp. 411-419, Feb 2011.

[4] B. Xu, A. Oudalov, J. Poland, A. Ulbig, and G. Andersson, "BESS control strategies for participating in grid frequency regulation," IFAC Proceedings Volumes, vol. 47, no. 3, pp. 4024-4029, Aug 2014.

[5] V. Gevorgian, Y. Zhang, and E. Ela, "Investigating the impacts of wind generation participation in interconnection frequency response," IEEE Transactions on Sustainable Energy, vol. 6, no. 3, pp. 1004-1012, Jul 2015.

[6] S. V. Dhople, S. S. Guggilam, and Y. C. Chen, "Linear approximations to AC power flow in rectangular coordinates," in 53rd Annual Allerton Conference on Communication, Control, and Computing, 2015, pp. 211-217.

[7] S. S. Guggilam, E. Dall'Anese, Y. C. Chen, S. V. Dhople, and G. B. Giannakis, "Scalable optimization methods for distribution networks with high PV integration," IEEE Transactions on Smart Grid, vol. 7, no. 4, pp. 2061-2070, Jul 2016.

[8] V. Donde, M. A. Pai, and I. A. Hiskens, "Simulation and optimization in an AGC system after deregulation," IEEE Transactions on Power Systems, vol. 16, no. 3, pp. 481-489, Aug 2001.

[9] D. Apostolopoulou, P. W. Sauer, and A. D. Domìnguez-Garcìa, "Automatic generation control and its implementation in real time," in 47th Hawaii International Conference on System Sciences, 2014, pp. 2444-2452.

[10] V. A. K. Pappu, B. Chowdhury, and R. Bhatt, "Implementing frequency regulation capability in a solar photovoltaic power plant," in Proceedings of North American Power Symposium, Arlington, Texas, USA, 2010, pp. 1-6.

[11] A. Hoke and D. Maksimović, "Active power control of photovoltaic power systems," in Proceedings of IEEE Conference on Technologies for Sustainability, Portland, OR, USA, 2013, pp. 70-77.

[12] P. Mercier, R. Cherkaoui, and A. Oudalov, "Optimizing a battery energy storage system for frequency control application in an isolated power system," IEEE Transactions on Power Systems, vol. 24, no. 3, pp. 1469-1477, Aug 2009.

[13] S.-J. Lee, J.-H. Kim, C.-H. Kim, S.-K. Kim, E.-S. Kim, D.-U. Kim, K. K. Mehmood, and S. U. Khan, "Coordinated control algorithm for distributed battery energy storage systems for mitigating voltage and frequency deviations," IEEE Transactions on Smart Grid, vol. 7, no. 3, pp. 1713-1722, May 2016.

[14] D. Hammerstrom, J. Brous, D. Chassin et al., "Pacific Northwest GridWise testbed demonstration projects, part II: Grid Friendly Appliance project," Pacific Northwest National Laboratory, Richland, WA, USA, Tech. Rep. PNNL-17079, 2007.

[15] U. K. Market Transformation Program, "Dynamic demand control of domestic appliances," Market Transformation Programme, Tech. Rep., 2008.

[16] P. J. Douglass, R. Garcia-Valle, P. Nyeng, J. Ostergaard, and M. Togeby, "Smart demand for frequency regulation: Experimental results," IEEE Transactions on Smart Grid, vol. 4, no. 3, pp. 1713-1720, Sep 2013.
[17] M. Donnelly, D. Harvey, R. Munson, and D. Trudnowski, "Frequency and stability control using decentralized intelligent loads: Benefits and pitfalls," in Proceedings of IEEE Power and Energy Society General Meeting, Minneapolis, MN, USA, 2010, pp. 1-6.

[18] W. H. Kersting, Distribution System Modeling and Analysis. 2nd ed., Boca Raton, FL: CRC Press, 2007.

[19] C. Coffrin, D. Gordon, and P. Scott, "NESTA, the NICTA energy system test case archive," arXiv preprint arXiv:1411.0359, 2014.

[20] (2016) IEEE Power and Energy Society distribution test feeders. [Online]. Available: https://ewh.ieee.org/soc/pes/dsacom/testfeeders/.

[21] J. Chow and G. Rogers, Power System Toolbox. Cherry Tree Scientific Software, 2000. 